\title{
Local excision of rectal cancer after chemoradiation: feasibility depends on the primary stage
}

\author{
J. W. A. Burger • E. J. R. de Graaf • \\ P. G. Doornebosch • D. J. Grünhagen • K. Biermann • \\ J. H. de Wilt $\cdot$ C. Verhoef
}

Accepted: 11 February 2010 / Published online: 9 March 2010

(C) The Author(s) 2010. This article is published with open access at Springerlink.com

\section{Dear Editor,}

In the Netherlands, the standard treatment for T2-3 rectal cancer is a short-course preoperative radiotherapy $(5 \times 5 \mathrm{~Gy})$ followed by total mesorectal excision (TME). The Dutch TME trial proved that the addition of preoperative radiotherapy to surgery decreased the 5-year local recurrence rate from $11 \%$ to $6 \%$. For patients with locally advanced rectal cancer, a long course of preoperative chemoradiation therapy is usually performed $(25 \times 2$ Gy). This treatment is based on trials by Bosset and Gerard that demonstrated that the addition of 5-fluorouracil yielded higher response rates and lower local recurrence rates.

The introduction of neoadjuvant chemoradiation therapy for locally advanced rectal cancer has also introduced a dilemma. In approximately $10-15 \%$ of patients, a pathological complete response is seen in the resection specimen. For these patients, a wait-and-see policy may be beneficial, with regard to both survival and quality of life. However,

J. W. A. Burger $(\bowtie) \cdot$ C. Verhoef

Department of Surgical Oncology, Erasmus MC-Daniël den Hoed Cancer Center,

P.O. Box 2040, 3000 CA Rotterdam, The Netherlands

e-mail: j.burger@erasmusmc.nl

E. J. R. de Graaf • P. G. Doornebosch • D. J. Grünhagen

Department of Surgery, IJsselland Ziekenhuis,

P.O. Box 690, 2900 AR Capelle aan den IJssel,

The Netherlands

\section{K. Biermann}

Department of Pathology, Erasmus MC—Daniël den Hoed

Cancer Center,

P.O. Box 2040, 3000 CA Rotterdam, The Netherlands

J. H. de Wilt

Department of Surgical Oncology, UMC St. Radboud,

P.O. Box 9101, 6500 HB Nijmegen, The Netherlands preoperative staging of rectal carcinoma after chemoradiation therapy has proven to be inaccurate. Both Hiotis and Zmora showed that only $25-33 \%$ of rectal cancers that had a complete clinical response indeed had a complete pathological response. Many surgeons therefore choose to remove the tumour area by local excision to confirm the response to chemoradiation therapy pathologically. In a study by Guerrieri, 196 rectal cancer patients were treated by neoadjuvant radiation therapy and local excision alone. Excellent results were reported, even though these surgeons never performed salvage TME, irrespective of the pathological T-stage.

Hereby, we present the case of a patient with a clinical T3-4N0 rectal cancer and a clinical complete response to chemoradiation therapy that was treated by local excision. This 74-year-old woman was seen in the outpatient department with complaints of rectal blood loss and residual stool. Rectal examination revealed a ventral tumour, located just above the anal verge. The tumour appeared mobile, and the vaginal mucosa was intact. At colonoscopy, a biopsy was taken, revealing a moderately differentiated adenocarcinoma. A magnetic resonance imaging (MRI) of the pelvis showed rectal cancer, extending 5-10 $\mathrm{cm}$ from the anal verge. The carcinoma invaded the subserosa and perirectal tissue. Ingrowth in the vaginal wall could not be ruled out. There was no lymphadenopathy, and no distant metastases were found after computed tomography of the abdomen and thorax. The clinical tumour, node, and metastasis (TNM) stage was cT3-4N0M0.

The patient was scheduled for neoadjuvant chemoradiation therapy $(25 \times 2 \mathrm{~Gy}+$ capecitabine 2 bid $825 \mathrm{mg})$. Six weeks after chemoradiation, the response was evaluated by rectal examination, MRI of the pelvis, endoanal ultrasound, and rectoscopy. There appeared to be a complete response, without lymphadenopathy. Only a small 
ulcer remained, located centrally in the area where the tumour was located. Again, no distant metastases were seen. After discussing the results with the patient, she was scheduled for local excision of the tumour area by TEM 10 weeks after completion of chemoradiation therapy, although the tumour area had not been tattooed prior to chemoradiation therapy. The central ulcer with surrounding fibrosis was excised with a margin of $1 \mathrm{~cm}$ macroscopically normal mucosa, resulting in a specimen with an area of $6 \times$ $5 \mathrm{~cm}$. Standardised pathology revealed a moderately differentiated adenocarcinoma, infiltrating the muscularis propria. The area of the ulcer and surrounding fibrosis was $3 \times 2.5 \mathrm{~cm}$. The margins were complete with a minimal distance of $4 \mathrm{~mm}$, resulting in a R0 ypT2Nx resection.

A salvage TME was proposed, with which the patient agreed. Since she experienced soiling before treatment, we decided to perform an intersphincteric rectal amputation rather than making a low anastomosis. The procedure took place 18 weeks after the completion of chemoradiation therapy. The rectum was found to be fixed to the posterior vagina. The posterior vaginal wall was partially excised, and the defect closed with sutures.

The pathology report revealed extensive radiation effects and scar tissue. Notably, a focus of vital adenocarcinoma was found. It was located in the perirectal fat and had a diameter of $2 \mathrm{~mm}$. Four perirectal lymph nodes without tumour were found. The margins were complete. As a consequence, the pathological TNM stage after chemoradiation therapy was changed to ypT3N0 rectal cancer.

Although this 74-year-old patient had a clinical complete response to neoadjuvant chemoradiation, pathological examination of the specimen revealed a $\mathrm{T} 2$ rectal cancer with complete margins after local excision. Notably, the salvage TME specimen contained a viable cancer cell nest at a deeper level, changing the pathological $\mathrm{T}$ stage to $\mathrm{T} 3$ rectal cancer. To our knowledge, no similar case has been reported. We argue that the local excision of rectal cancer after chemoradiation carries the risk of leaving behind viable cancer cell nests. This is probably caused by a scattered regression pattern of rectal cancer in response to chemoradiation therapy. With regard to local recurrence, it is often thought that the local recurrence after a local excision is caused by a concurrent lymph node disease. However, failure to remove viable cancer cell nests is likely to play a role in local recurrence as well. Because resection margins may be complete, these viable cancer cell nests can easily go unnoticed.

Apart from increasing the risk of local recurrence, the remaining viable cancer cell nests can also result in pathological understaging, as was the case in this patient. The pathological $\mathrm{T}$ stage after chemoradiation therapy and local excision of rectal cancer determines the risk of local recurrence and lymph node involvement. It is the basis on which surgeons decide to either adopt an observational strategy or proceed with a salvage TME. Borschitz and Bujko reported that local excision of ypT0 rectal cancer is associated with a $0 \%$ local recurrence rate and a $5 \%$ risk of lymph node disease. YpT1 has a 2\% local recurrence rate and an $8 \%$ risk of lymph node disease. Therefore, ypT0 and ypT1 are often seen as candidates for an observational strategy. On the other hand, ypT2 is associated with a 7\% local recurrence rate and a $28 \%$ risk of lymph node disease. YpT3 is associated with a $21 \%$ local recurrence rate and a $55 \%$ risk of lymph node disease. Local excision of ypT2 or ypT3 rectal cancer is therefore usually followed by a salvage TME. It follows that an accurate pathological staging after local excision is of the utmost importance.

To avoid this kind of irradical resection, local recurrence, and pathological understaging after local excision of rectal cancer, it is imperative that all tissues that were primarily invaded by the rectal cancer are removed. The circumferential mucosal margins of the tumour should be marked by tattooing the edges of the tumour prior to the start of neoadjuvant chemoradiation therapy. The deep margins cannot be marked. This means that when the primary $\mathrm{T}$ stage was cT3, a complete resection of the mesorectal layer deep to the tattooed tumour area is mandatory. Moreover, the accuracy of preoperative assessment of the depth of invasion is limited. Therefore, mesorectal excision may also be advisable for cT2 rectal cancer. Although mesorectal excision by local techniques is not a standard procedure, it is feasible, both by TEM and other endoscopic techniques. It is important to realise that local excision of all tissues that were primarily invaded by rectal cancer is not feasible if the primary stage was T4. Moreover, local mesorectal excision does not result in an adequate lymphadenectomy.

\section{Dear Editor,}

We agree that the results of local excision of rectal cancer after chemoradiation therapy are promising. However, the case we reported in this letter proves that an accurate pathological staging can only be achieved if all tissues that were primarily invaded by the tumour are excised. Therefore, circumferential margins of the tumour should be tattooed prior to chemoradiation therapy, and in patients with cT3 rectal cancer, a complete excision of the mesorectal tissue deep to the tumour area is mandatory. Patients with cT4 rectal cancer or lymph node-positive disease are not good candidates for local excision.

Open Access This article is distributed under the terms of the Creative Commons Attribution Noncommercial License which permits any noncommercial use, distribution, and reproduction in any medium, provided the original author(s) and source are credited. 